\section{P4.49 ADOLESCENTS KNOWLEDGE AND AWARENESS CONCERNING HIVIAIDS AND FACTORS AFFECTING THEM IN DONYO SABUK, KENYA}

Jacquiline Nyambura Njeru. Mukunike CBO, Donyo Sabuk, Kenya

10.1136/sextrans-2017-053264.546

Introduction Adolescents are more vulnerable than adults on sexually transmitted diseases and HIV/AIDS. Among the adolescents, girls are more vulnerable to STDs including HIV/ AIDS. Their knowledge about STDs and HIV/AIDS is very poor. This study was conducted between February 2016 and September 2016 investigating adolescent's between 10-19 years knowledge about sexually transmitted diseases including HIV/AIDS, its mode of transmission and prevention methods.

Method A multistage cluster sampling technique was used to select the sample. Data on 300 upper primary school female adolescents and 300 high school female adolescents was analysed. The study found that a large proportion of upper primary school adolescents ages between10-14 had very little knowledge on sexually transmitted diseases and HIV. More than three quarter of high school adolescents ages 15-19 years had knowledge about HIV respectively. On an average, about one third of them had no knowledge on HIV in terms of modes of transmission and prevention.

Results The multivariate logistic regression analysis revealed that adolescent age, years of schooling and knowledge on STIs and STDs appeared to be important predictors of the awareness about HIV/AIDS.

Conclusion Useful and fruitful awareness campaigns to educate the upper primary female adolescents regarding the health consequences of STDs including HIV/AIDS and integrated approach is strongly suggested for creating knowledge and awareness to control the spread of HIV/AIDS among adolescents in Ndonyo Sabuk, Murang'a County, Kenya.

\section{P4.50 ASSOCIATIONS OF HIV TESTING WITH HIV STIGMA: IMPLICATIONS FOR FAITH BASED HIV TESTING AND TREATMENT}

${ }^{1}$ J.M Stewart, C Budhathoki,. ${ }^{2}$, D Bellinger,. ${ }^{3},{ }^{1}$ J.B Hamilton, . 'Johns Hopkins University School of Nursing, Department of Community Public Health, Baltimore, MD 21205, USA; ${ }^{2} J o h n s$ Hopkins University School of Nursing, Department of Acute and Chronic Care, Baltimore, MD 21205, USA; ${ }^{3}$ Johns Hopkins University School of Nursing, Baltimore, MD 21205, USA

\subsection{6/sextrans-2017-053264.547}

Introduction The promotion of HIV testing for African Americans is a key approach to reducing both the number of individuals that are unaware of their status as well as the transmission of HIV. African American churches can serve as alternative venues to promote HIV testing and linkage to care. However, HIV stigma serves as a barrier to the integration of HIV testing in churches. Results are mixed as to the levels of HIV stigma among African American churches.

Methods We surveyed the congregants of African American churches $(n=177)$ and compared participants from two types of churches, those with and without an HIV testing facility.
Results The HIV stigma score was not significantly different between congregants at churches with HIV testing and those without testing. However the participants from churches that did not offer HIV testing showed significantly different views on homosexuality, sexuality and drug abuse as barriers to HIV testing compared to those who attended churches that offered HIV testing $(\mathrm{p}<0.001)$.

Conclusion These results have implications for the important role that African American churches could have in not only offering HIV testing but reducing associated barriers.

\section{P4.51 ENGAGING YOUNG PEOPLE FROM MIGRANT AND REFUGEE BACKGROUNDS WITH SEXUAL AND REPRODUCTIVE HEALTH PROMOTION AND CARE IN SYDNEY, AUSTRALIA}

Jessica Botfield, Christy Newman, ZWI Anthony. UNSW Australia, Sydney, Australia

\subsection{6/sextrans-2017-053264.548}

Introduction Migrant and refugee young people are often underserved by mainstream sexual and reproductive healthcare $(\mathrm{SRH})$ in Australia and other high-income countries. Research is being undertaken to explore this, with particular emphasis on understanding the views and experiences of these young people regarding services for this aspect of health. In addition, we are exploring community, provider and policy perspectives on the complexities of engaging this diverse range of young people.

Methods A structured scoping review was undertaken to synthesise the contributions of research to understanding this topic and identify gaps in the literature. Semi-structured interviews were conducted with 23 key informants (health service providers, policymakers, academics, community advocates) to elicit their views on the complexities of, and best practice approaches to, engaging this group with SRH promotion and care. Semi-structured interviews with migrant and refugee young people are currently underway.

Results The scoping review identified two knowledge gaps: 1) perspectives of migrant and refugee young people regarding $\mathrm{SRH}$ health care, and 2) engagement of this group with SRH services. Key informant interviews revealed pivotal aspects of how professionals navigate this field: 1) appreciating the complexities of cultural diversity; 2) recognising structural barriers and disincentives to engagement; 3) normalising sexual health; 4) balancing 'youth-friendly' and 'culturally-competent' priorities; and 5) going beyond simple language translation to support open dialogue and meaningful engagement.

Conclusion As many high-income countries undergo demographic expansion and diversification due to migration, integrated and appropriate nation-wide frameworks for the design and delivery of SRH promotion and care is essential. The current phase of research seeks to document the views of migrant and refugee young people, aiming to capture both the synergies and divergences that are likely to be apparent between professional perspectives and the lived experience of this group of young people. 